\title{
Lineage tracing demonstrates the venous origin of the mammalian lymphatic vasculature
}

\author{
R. Sathish Srinivasan, ${ }^{1}$ Miriam E. Dillard, ${ }^{1}$ Oleg V. Lagutin, ${ }^{1}$ Fu-Jung Lin, ${ }^{2}$ Sophia Tsai, ${ }^{2,3}$ \\ Ming-Jer Tsai, ${ }^{2,3}$ Igor M. Samokhvalov, ${ }^{4}$ and Guillermo Oliver ${ }^{1,5}$ \\ ${ }^{1}$ Department of Genetics and Tumor Cell Biology, St. Jude Children's Hospital, Memphis, Tennessee 38105, USA; \\ ${ }^{2}$ Department of Molecular and Cellular Biology, Baylor College of Medicine, Houston, Texas 77030, USA; ${ }^{3}$ Developmental \\ Biology Program, Baylor College of Medicine, Houston, Texas 77030, USA; ${ }^{4}$ Laboratory for Stem Cell Biology, Center for \\ Developmental Biology, RIKEN Kobe, Kobe 650-0047, Japan
}

The origin of the mammalian lymphatic vasculature has been debated for more than 100 years. Whether lymphatic endothelial cells have a single or dual, venous or mesenchymal origin remains controversial. To resolve this debate, we performed Cre/loxP-based lineage-tracing studies using mouse strains expressing Cre recombinase under the control of the Tie2, Runx1, or Prox1 promoter elements. These studies, together with the analysis of Runx1-mutant embryos lacking definitive hematopoiesis, conclusively determined that from venous-derived lymph sacs, lymphatic endothelial cells sprouted, proliferated, and migrated to give rise to the entire lymphatic vasculature, and that hematopoietic cells did not contribute to the developing lymph sacs. We conclude that the mammalian lymphatic system has a solely venous origin.

[Keywords: Lymphatic endothelial cells; lymphangiogenesis; Prox1; mouse; lineage tracing; Runx1]

Supplemental material is available at http://www.genesdev.org.

Received June 29, 2007; revised version accepted August 10, 2007.

The lymphatic vasculature returns extravasated fluids to the blood circulation, thereby maintaining tissue fluid homeostasis. It also facilitates immune surveillance and lipid absorption from the intestine. Furthermore, the lymphatic vasculature is a major route for tumor metastasis (Oliver and Alitalo 2005). Recently, the identification of lymphatic markers has greatly increased our understanding of the genes and mechanisms that regulate the development of the lymphatic vasculature (lymphangiogenesis) (Oliver and Alitalo 2005). Despite this progress, our knowledge of the lymphatic system is still rudimentary, and some aspects remain unresolved.

A century-old debate persists about the origin of embryonic lymph sacs, the structures from which the lymphatic vasculature is derived. Studies performed in the early 1900s proposed that early during development, lymph sacs originate from budding venous endothelial cells (ECs); from these initial structures, the entire lymphatic system then spreads into surrounding tissues and organs (Sabin 1902). An alternative view proposed that lymph sacs arise from mesoderm-derived endothelial precursors and secondarily establish venous connections (Huntington and McClure 1910).

We previously showed that in mice, starting around

${ }^{5}$ Corresponding author.

E-MAIL guillermo.oliver@stjude.org; FAX (901) 526-2907.

Article is online at http://www.genesdev.org/cgi/doi/10.1101/gad.1588407. embryonic day 9.5 (E9.5), the homeobox gene Prox1 is expressed in a subpopulation of blood ECs (BECs) in the anterior cardinal vein (Wigle and Oliver 1999). On the basis of our expression and functional analyses, we favored Sabin's venous model and proposed that Prox1expressing ECs bud from the veins and form embryonic lymph sacs and the lymphatic vasculature (Wigle and Oliver 1999|. In addition, our finding that Prox1-null embryos are devoid of lymphatic vasculature (Wigle and Oliver 1999) due to a failure in lymphatic cell-type specification (Wigle et al. 2002) definitively determined the crucial role of Prox1 in developmental lymphangiogenesis. Although these initial studies demonstrated that Prox1 activity confers a lymphatic endothelial cell (LEC) phenotype on venous LEC progenitors (Oliver and Detmar 2002; Wigle et al. 2002), they did not determine the origin of the Prox1-expressing progenitors, nor did they exclude the possibility that sources other than Prox1expressing venous LEC progenitors contribute to mammalian lymphangiogenesis.

Recent work in different model organisms has provided mixed results about the origin of LECs. In chicken and frog embryos, LECs arise from venous-derived ECs and mesenchymal lymphangioblasts (Wilting et al. 2000, 2006; Ny et al. 2005). In zebrafish, time-lapse imaging revealed that LECs of the main thoracic duct-like vessel arise from primitive veins (Yaniv et al. 2006). However, 
this mosaic analysis did not determine whether the entire zebrafish lymphatic vasculature is solely venous derived or has other contributing sources (e.g., mesenchyme-derived lymphangioblasts). Furthermore, unlike mammals, zebrafish do not appear to have lymph sacs; thus, the critical steps leading to the formation of the entire lymphatic networks probably differ in these two model systems. In mammals, the current data propose that venous-derived LECs, hematopoietic cell-derived circulating endothelial progenitors (CEPs), and transdifferentiating leukocytes and macrophages are putative sources of LECs during embryonic and adult lymphangiogenesis (in health and disease) (Wigle and Oliver 1999; Wigle et al. 2002; Salven et al. 2003; Maruyama et al. 2005; Religa et al. 2005; Buttler et al. 2006; Kerjaschki et al. 2006; Sebzda et al. 2006). These results indicate that the source(s) of LECs and the mechanisms of lymphatic vasculature formation are species specific, and they highlight the importance of resolving this century-long question concerning the origin(s) of the lymphatic system to facilitate our understanding of normal and pathological lymphangiogenesis.

To this end, we used tamoxifen (TM)-inducible Cre/ LoxP-based tracing systems to genetically label early Prox1-expressing murine LECs and determine their origin and fate. Fate-mapping studies were also performed to evaluate the contribution(s) of venous ECs and hematopoietic cells to the developing lymphatic vasculature and to elucidate the stepwise mechanisms of lymphangiogenesis.

\section{Results}

Generation of the Prox1-CreER ${ }^{\mathrm{T} 2}$ mouse strain

In the mouse, Prox 1 expression in ECs initiates around E9.5 in the anterior cardinal vein (Wigle and Oliver 1999). To irreversibly mark and follow the fate of Prox $1^{+}$ cells that contribute to developmental lymphangiogenesis, we genetically labeled Prox1-expressing cells with a TM-inducible Cre-ER $R^{T 2} /$ LoxP-based tracing system (Danielian et al. 1998; Indra et al. 1999). Gene targeting inserted Cre-ER ${ }^{T 2}$ into the mouse Prox1 locus (Supplementary Fig. 1) to generate the Prox1-CreER ${ }^{T 2}$ allele. To generate the targeting construct and avoid the haploinsufficiency phenotype observed in Prox $1^{+/ L a c Z}$ mice (Wigle and Oliver 1999; Harvey et al. 2005), we inserted at the second intron of Prox 1 a cassette containing a fusion of a synthetic splice acceptor site, a fragment of Prox 1 cDNA containing exons 3 and 4, an internal ribosome entry site, Cre-ER $R^{T 2}$, and a poly(A) transcriptionstop signal (Supplementary Fig. 1). This modified Prox1 allele should express Prox1 and Cre-ER ${ }^{T 2}$ from a single bicistronic transcript, thereby recapitulating the normal pattern of Proxl expression. Targeted embryonic stem cells were used to generate the Prox1-CreER ${ }^{T 2}$ mouse strain. As expected, mice that were either heterozygous or homozygous for the modified Prox 1 allele were viable and showed no obvious phenotypic alteration.

To evaluate whether the modified allele functioned as expected, we used the $R 26 R$ reporter line (Soriano 1999) to monitor the activation of $\beta$-galactosidase (lac $Z$ ) in descendants of Prox1-expressing cells at different time points. No lacZ labeling was detected prior to TM administration (data not shown); instead, most Prox1-expressing cells were $1 a c Z^{+}$after TM exposure (Supplementary Fig. 2).

Next, we determined the optimal TM dose required to visualize the progeny of Proxl-expressing cells in the developing lymphatics. We determined that intraperitoneal injection of 3-5 mg of TM per $40 \mathrm{~g}$ of body weight into pregnant dams was sufficient to label embryonic cells without affecting embryonic viability. As expected, the higher the dose of TM, the more efficient and rapid the extent of cell labeling (data not shown). Accordingly, our choice of TM dose depended on the goal of the particular experiment: To label the maximum number of LECs, we used $5 \mathrm{mg}$ of TM, and to label the Prox1-expressing LEC lineage within the narrowest window of time, we used $3 \mathrm{mg}$ of TM. Therefore, in an initial experiment to determine whether the lymphatic expression of Prox1-CreER ${ }^{\mathrm{T} 2}$ recapitulates that of endogenous Prox1, we exposed Prox1-CreER ${ }^{T 2} ; R 26 R$ embryos to 5 $\mathrm{mg}$ of TM at different embryonic stages and compared their X-gal expression patterns with those of comparably staged control Prox $1^{+/ L a c Z}$ embryos (Supplementary Fig. 2).

In addition to the developing lymphatics, we also determined the extent of lacZ labeling in other Prox1-expressing embryonic cell types (data not shown). With the exception of the heart and CNS, where only a few lac $Z^{+}$ cells were detected, all other cell types appeared to faithfully recapitulate the Proxl expression profile (Supplementary Fig. 2; data not shown). Similar to what was reported for other inducible Cre strains (Dor et al. 2004; Zhang et al. 2005), the efficiency of cell labeling was variable. Therefore, the pattern of Proxl expression was mosaic in the generated Prox1-CreER ${ }^{T 2}$ mouse strain. This feature was most likely caused by multiple factors such as small experimental variations in the effective dose of TM available at any certain time to any particular cell of interest, variations in the susceptibility of the specific locus, and the transient nature of the cell's access to TM. Nevertheless, the labeled domain was reproducible with only small variations in the percentage of marked cells. The reduced number of $1 a c Z^{+}$cells detected in the heart and CNS could be explained by the fact that most Prox1-expressing cells in those tissues are post-mitotic.

Having determined the optimal dose of TM, we next performed detailed cell-labeling time-course studies to determine the kinetics of cell labeling mediated by Prox1-CreER ${ }^{T 2}$. We have previously shown that Prox1expressing LECs are normally detected in and near the cardinal vein at around E9.5 (Wigle and Oliver 1999). Considering 9 a.m. of the day the vaginal plug was detected as E0.5, we have now precisely determined that Proxl expression initiates in the anterior cardinal vein at E9.75. No lacZ $^{+}$LECs were detected in E10.5 embryos exposed to $3 \mathrm{mg}$ of TM that was administered to pregnant Prox1-CreER $R^{T 2} \times R 26 R$ females at E9.0 (Supple- 
mentary Fig. 3A). This finding suggested that $3 \mathrm{mg}$ of TM mediates Cre-ER ${ }^{\mathrm{T} 2}$-mediated cell labeling for $<24 \mathrm{~h}$. Instead, $1 a c Z^{+}$LECs were detected in and around the cardinal vein of E10.5 embryos exposed to TM at E9.5 (Supplementary Fig. 3B,C, arrows). Next, to precisely determine the kinetics of Cre-mediated $R 26 R$ activation, we administered $3 \mathrm{mg}$ of TM at E10.5, when Proxl is strongly expressed in the cardinal vein, and isolated embryos 4, 6, 8, and $12 \mathrm{~h}$ later. The lac $\mathrm{Z}^{+}$LECs were first detected $6 \mathrm{~h}$ after TM exposure; labeling was found in and near the cardinal vein (Supplementary Fig. 3D; data not shown).

\section{Venous ECs are the earliest Prox1-expressing LEC progenitors}

An initial step in developmental lymphangiogenesis is the formation of primitive lymph sacs. Based on expression analysis, we previously proposed that in mice these sacs arise from Prox $1^{+}$LECs located in the cardinal veins (Wigle and Oliver 1999). Therefore, we first wanted to conclusively determine whether mammalian primitive lymph sacs are formed by Prox1-expressing venous ECs. To do this, we first aimed to exclusively label the earliest Prox $1^{+}$LECs located in the anterior cardinal vein at E9.75. We performed a kinetic analysis (similar to the one described above) by exposing embryos to $3 \mathrm{mg}$ of TM at E9.5 (6 $\mathrm{h}$ prior to the first appearance of Prox $1^{+}$ECs in the anterior cardinal vein). In agreement with the above results, we first detected scattered lac $Z^{+}$LECs in some embryos $12 \mathrm{~h}$ after TM administration (or $6 \mathrm{~h}$ after Prox1-CreER ${ }^{T 2}$ locus expression); by $24 \mathrm{~h}$ and once TM was no longer active, $\operatorname{lac}^{+}$LECs were detected in or near the cardinal vein in all embryos (Supplementary Fig. 3C,E). These results demonstrate that administration of $3 \mathrm{mg}$ of TM at E9.5 exclusively labels the earliest Prox $1^{+}$ LECs located in the embryonic veins.
Next, for fate-mapping experiments, we administered $3 \mathrm{mg}$ of TM at E8.5 or E9.5 to Prox1-CreER ${ }^{T 2}$ mice to activate the $R 26 R$ reporter. The earliest contribution of Proxl-expressing cells to the developing lymphatics was assessed by the presence of $1 a c Z^{+}$cells in the forming jugular lymph sacs and peripheral (superficial) lymphatics of E13.5 embryos. No lacZ $Z^{+}$cells were detected in these structures when TM was administered at E8.5 (Fig. $1 \mathrm{~A}, \mathrm{D})$; scattered $\mathrm{lac}^{+}$cells were seen only in the liver (Fig. 1A). However, lac $Z^{+}$cells were detected in those lymphatic regions when TM was administered at E9.5 (Fig. 1B,E). These results indicate a relation between the initiation of Prox 1 expression in the anterior cardinal vein and the formation of the primitive lymph sacs. They argue that ECs in the anterior cardinal veins at around E9.75 are the earliest source of Prox1-expressing LEC progenitors that contribute to the developing lymph sacs and peripheral lymphatics.

The LEC identity of the lacZ-labeled cells was determined using appropriate molecular markers (Supplementary Fig. 4). The LEC identity of $1 a c Z^{+}$cells agreed with our earlier characterization of Proxl expression during developmental lymphangiogenesis (Wigle and Oliver 1999; Wigle et al. 2002). At E13.5, the number of $1 a c Z^{+}$ cells in the peripheral lymphatics and lymph sacs increased with the sequential administration of TM at later developmental stages (Fig. 1C,F), which supports the proposal that embryonic lymphangiogenesis requires a continuous source of Prox $1^{+}$venous LEC progenitors. Furthermore, in agreement with van der Putte (1975), who proposed that lymphatic structures develop in a temporal rostrocaudal manner, our analysis of E13.5 embryos showed that TM exposure at E9.5 labeled lac $^{+}$ LECs mostly in the anterior part of the embryo (Fig. 1B, arrow); TM exposure at E10.5 labeled these cells as well as cells around the caudal perimesonephric region (Fig. $1 \mathrm{C}$, arrowhead).
Figure 1. Venous ECs are the earliest LEC progenitors. Prox1-CreER ${ }^{T 2}$;R26R pregnant dams were injected with $3 \mathrm{mg}$ of TM at different times and X-gal-stained at E13.5. (A) TM administration at E8.5 labeled Prox 1 descendent cells only in the liver (arrow); no lac $Z^{+}$LECs were detected in superficial lymphatics or lymph sacs (shown in $D$ ). (B) Following TM administration at E9.5, the first superficial lac $Z^{+}$ LECs were identified around the jugular region (arrow) and the jugular lymph sacs (shown in $E$ ). (C) Upon TM administration at E10.5, the number of $1 a c Z^{+}$LECs increased in the jugular region (arrow) and extended toward the periorbital region /red arrowhead) and the perimesonephric region (black arrowhead). (F) The number of lac $Z^{+}$LECs in the jugular lymph sacs also increased. Bar, $100 \mu \mathrm{m}$.
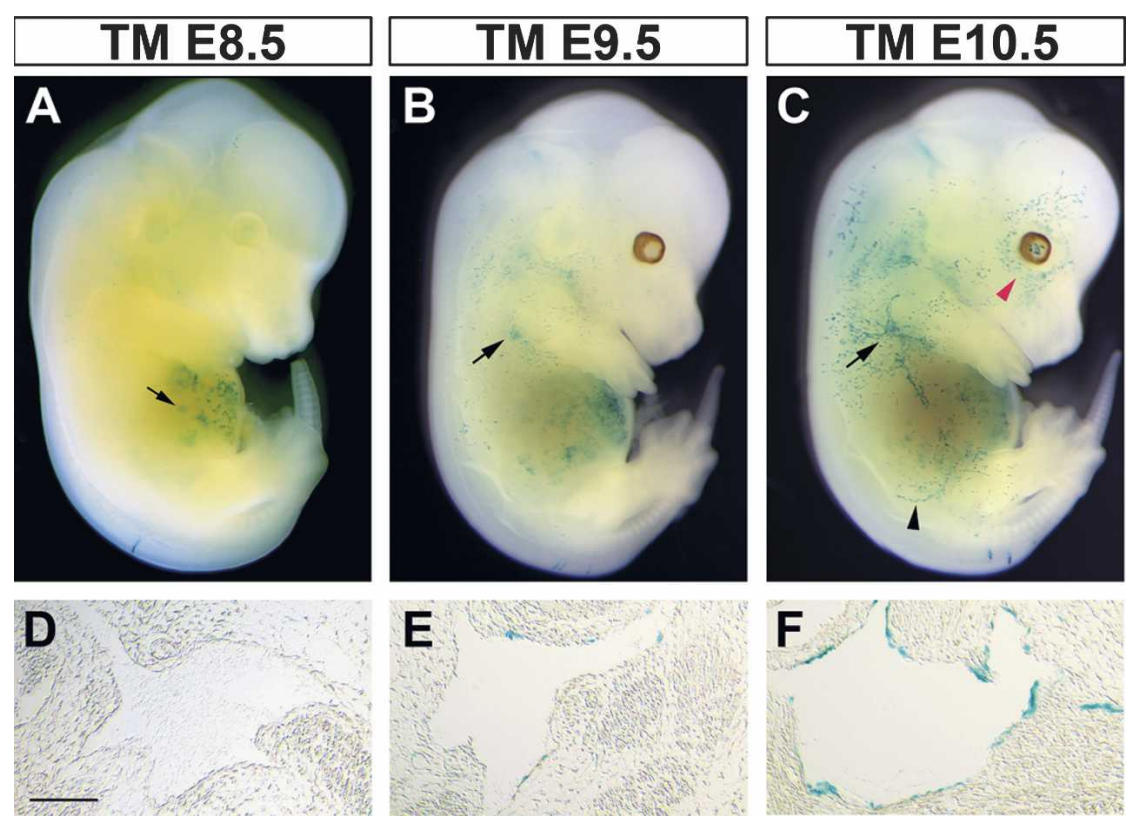
These results support the proposal that in the developing mouse embryo, Prox $1^{+}$LEC progenitors are not present prior to the appearance of the Proxl-expressing venous ECs in the anterior cardinal vein (around E9.75). In addition, sequential, stage-dependent administration of TM correlates with an increasing number of $l a c Z^{+}$ cells in the lymph sacs and peripheral lymphatics, suggesting that ECs in the cardinal veins are a continuous source of Proxl-expressing LECs, at least until the lymph sacs form.

The lymphatic vasculature arises by sprouting, proliferation, and migration of LECs

Once we determined that Proxl-expressing venous ECs are the earliest LEC progenitors contributing to the developing lymph sacs and peripheral lymphatics, we aimed to assess the mechanisms that control LEC sprouting into peripheral tissues, thereby giving rise to the whole lymphatic vasculature. To do this, we performed similar lineage-tracing studies using Prox1$\operatorname{CreER}^{T 2}$;R26R embryos. TM (5 mg) remains active for $<2.5 \mathrm{~d}$; therefore, to efficiently label as many Prox $1^{+}$cells as possible in the forming lymph sacs, TM was administered at E10.5, and embryos were isolated and analyzed daily between E11.5 and E15.5.

At E11.5, most superficial lac $Z^{+}$cells were located anterior to the developing forelimbs; a few were scattered more caudally (Fig. 2A). Most $1 a c Z^{+}$cells were LECs that had originated from the anterior (Supplementary Fig. 5A) and posterior cardinal veins and the iliac veins (data not shown). Therefore, these $1 a c Z^{+}$cells were the earliest descendants of Prox1-expressing venous ECs. At E12.5, the number of $1 a c Z^{+}$cells along the anteroposterior axis had increased (Fig. 2B), and the cells were easily detected in the lymph sacs (Supplementary Fig. 5B). At E13.5, $1 a c Z^{+}$cells were sprouting from the lymph sacs (Supplementary Fig. 5C). Many more $1 a c Z^{+}$descendents were observed around the jugular, perimesonephric, and eye regions (Fig. 2C), where they form the rostral periorbital lymphatics. Later the number of superficial lac $^{+}$cells derived from Prox1-expressing E10.5 progenitors increased substantially, particularly around the jugular and perimesonephric regions (Fig. 2D,E), and the expression pattern resembled that of E15.5 Prox $1^{+/ L a c Z}$ embryos (Fig. 2F). The steady expansion of $l a c Z^{+}$cells along the anteroposterior axis is consistent with the continuous sprouting, proliferation, and migration of LECs from the lymph sacs.

Next, we determined the spatiotemporal contribution of LEC progenitors to the deeper lymphatic plexus. First, and in order to label the maximum number of LECs in the deeper lymphatics, Prox1-CreER ${ }^{T 2} ; R 26 R$ embryos were exposed to $5 \mathrm{mg}$ of TM at E10.5, and their lungs and intestines were isolated and X-gal-stained at E16.5. The $1 a c Z^{+}$cells were detected in the lungs and mesenteric lymphatics (Fig. 3B,F) in a pattern similar to that in Prox $1^{+/ L a c Z}$ embryos (Fig. 3A,E). We speculated that like superficial lymphatics (Fig. 1), the appearance of the deeper lymphatics also follows an anteroposterior, spatiotemporal order. To test this possibility, we exposed Prox1-CreER ${ }^{T 2} ; R 26 R$ embryos to $3 \mathrm{mg}$ of TM (to label Prox $1^{+}$cells within a 24 -h time frame) at either E9.5 or E10.5; their lungs and mesenteries were X-gal-stained at E16.5. As previously explained, $3 \mathrm{mg}$ of TM less efficiently, and therefore less intensely, labeled Prox $1^{+}$LECs than did the 5-mg dose; however, we observed $\mathrm{LacZ}^{+}$ LECs in the lungs of E16.5 embryos that were exposed to $3 \mathrm{mg}$ of TM at either E9.5 (Fig. 3C) or E10.5 (Fig. 3D). It
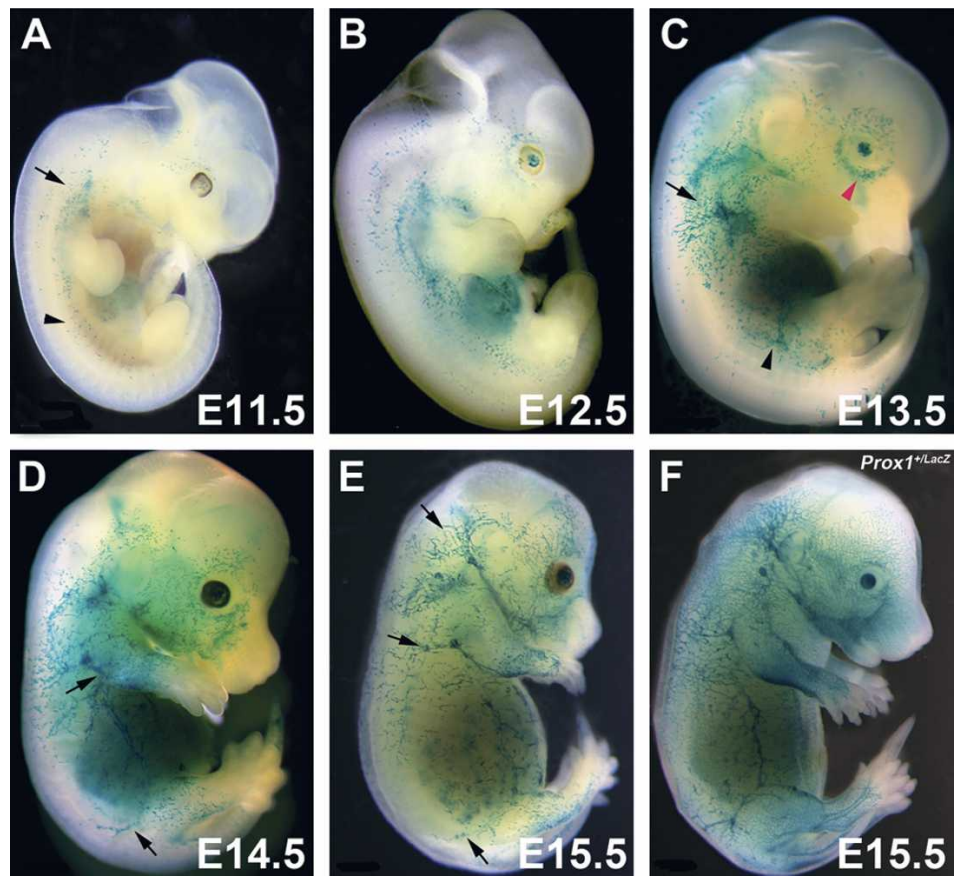

Figure 2. The superficial lymphatic vasculature arises by continuous sprouting, proliferation, and migration of LECs. Prox1-CreER ${ }^{T 2} ; R 26 R$ pregnant dams were injected with $5 \mathrm{mg}$ of TM at E10.5, and embryos were analyzed by X-gal staining at later time points. (A) At E11.5, most lacZ $^{+}$LECs are anterior to the forelimb (arrow); however, cells are also scattered along the anteroposterior axis (arrowhead). (B) At E12.5, the number of $l a c Z^{+}$LECs has uniformly increased along the embryonic axis. $(C)$ At E13.5, the largest accumulations of lac $Z^{+}$LECs are seen around the jugular (black arrow), perimesonephric (black arrowhead), and periorbital (red arrowhead) regions. $(D)$ At E14.5, the initial lymphatic network appears to sprout, and more superficial lac $Z^{+}$ LECs are present in the jugular and perimesonephric regions (arrows). (E) At E15.5, the pattern of superficial lymphatics appears identical to the one seen in similarly staged Prox $1^{+/ L a c Z}$ embryos (shown in F). Lymphatic vessel sprouting can be clearly seen (arrows). 

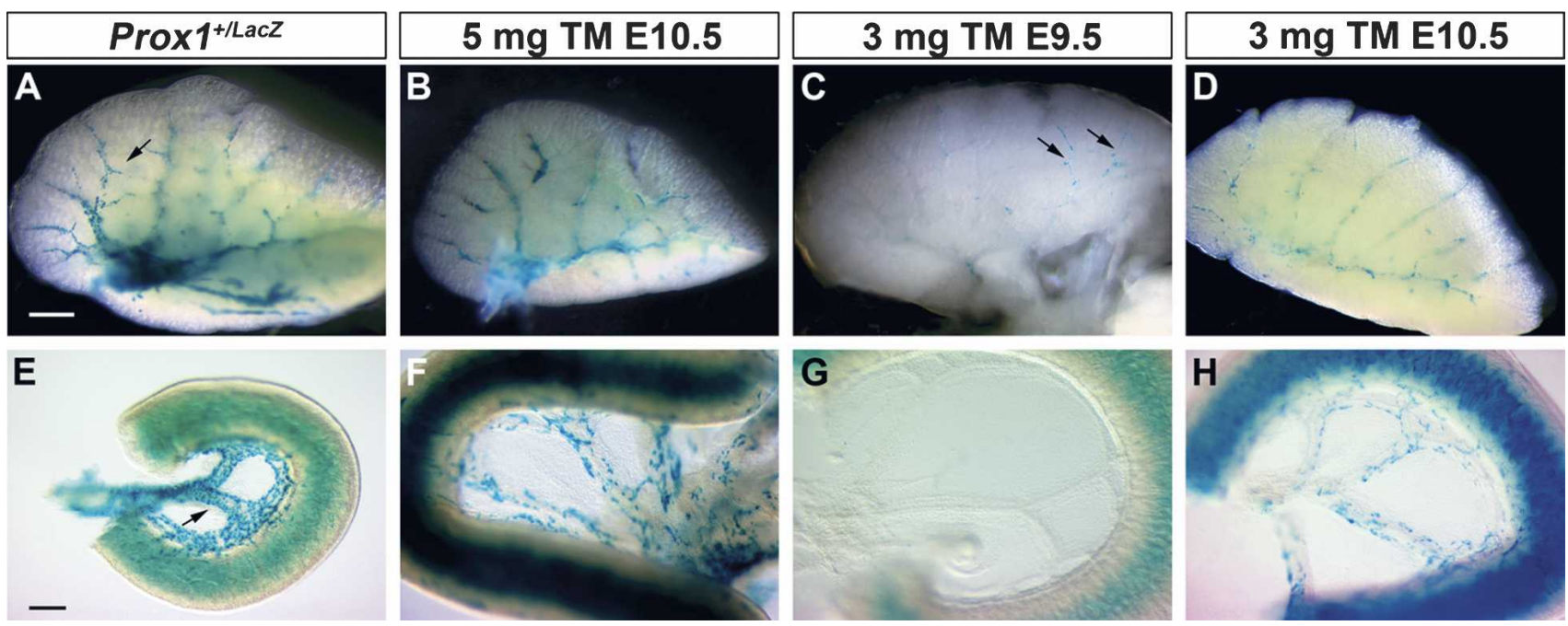

Figure 3. The deeper lymphatic vasculature arises by continuous sprouting, proliferation, and migration of LECs. Prox1$\operatorname{CreER}^{T 2} ; R 26 R$ pregnant dams were injected with 3 or $5 \mathrm{mg}$ of TM at $\mathrm{E} 9.5$ or E10.5, and the lungs and mesentery were analyzed by X-gal staining at E16.5. The normal distribution of lac $Z^{+}$LECs (arrows) in the lung $(A)$ and mesentery $(E)$ of an E16.5 Prox $1^{+/ L a c Z}$ embryo is shown. Blue staining inside the intestine reflects background trapping. This X-gal staining pattern is similar to the one observed in the lung $(B)$ and mesentery $(F)$ of E16.5 Prox1-CreER ${ }^{T 2} ; R 26 R$ embryos exposed to $5 \mathrm{mg}$ of TM at E10.5, which demonstrates that E10.5 Prox $1^{+}$LEC progenitors contribute to the deeper lymphatic vasculature. (C) After reduced TM ( $\left.3 \mathrm{mg}\right)$ exposure at E9.5, lac $Z^{+}$LECs are still detected (although their number is reduced) in the lungs of E16.5 Prox1-CreER ${ }^{T 2} ; R 26 R$ embryos (arrows). (G) No lacZ $Z^{+}$LECs are detected in the mesentery. An increase in the number of lac $Z^{+}$LECs can be seen in the lungs $(D)$ and mesentery $(H)$ of E16.5 Prox1-CreER ${ }^{T 2} ;$ R26R embryos exposed to $3 \mathrm{mg}$ of TM at E10.5. Bars: $A-D, 500 \mu \mathrm{m} ; E-H, 200 \mu \mathrm{m}$.

is worth mentioning that the lungs of embryos exposed to TM at E10.5 contained more LacZ $Z^{+}$LECs (Fig. 3D) than did those exposed at E9.5. This result supports our previous results (Fig. 1) indicating that ECs in the cardinal veins are a continuous source of Prox $1^{+}$LECs. On the other hand, in the analyzed mesenteric lymphatics, we observed $\mathrm{LacZ}^{+}$LECs only in E16.5 embryos exposed to TM at E10.5 (Fig. 3G,H). Together, these results support the proposal that rostral deep lymphatics appear first and are derived from rostrally located LEC progenitors, and caudal deep lymphatics form later from posteriorly located LEC progenitors.

The rostral jugular lymph sacs near the junction of the subclavian and anterior cardinal veins were the first to arise from the earliest Prox $1^{+}$LEC progenitors in the anterior cardinal vein at around E9.75. In contrast, LEC progenitors of the more posterior lymph sacs near the junction of the primitive iliac veins and the posterior cardinal veins appeared around E10.5 (Fig. 1). We have now demonstrated that local lymph sacs are the source of LECs that by sprouting, proliferation, and migration give rise to the lymphatic vasculature of nearby tissues and organs. Jugular lymph sacs form earlier and give rise to the lymphatics of the lungs and most likely other nearby tissues; posterior lymph sacs form later and give rise to the lymphatics of the mesentery and posterior organs. These lineage-tracing results support earlier anatomical descriptions (Sabin 1904; Heuer 1909); i.e., from the jugular lymph sacs, lymphatic capillaries spread toward anterior regions (head, neck, arms, and thorax), and from the posterior lymph sacs, lymphatics spread to posterior regions (abdominal wall, pelvis, and legs).

\section{Lymphatic vasculature is of venous origin}

Due to the mosaic labeling observed when using the Prox1-CreER ${ }^{T 2}$ mouse strain, we cannot exclude the possibility that other sources contribute to the formation of the lymph sacs. To conclusively demonstrate that mammalian lymph sacs and, therefore, the whole lymphatic vasculature are exclusively venous derived, we complemented the studies presented above by performing similar analyses using the available mouse strains Tie2-Cre, Prox $1^{\text {flox } /+}$, Coup-TFII $I^{\text {flox } /+}$, Runx1-MER-Cre-MER, and Runx1.

Tie2-Cre mice Tie2 is a receptor tyrosine kinase expressed in BECs and hematopoietic cells (Sato et al. 1993; Takakura et al. 1998). The interaction of Tie2 with its ligand angiopoietin-1 (Ang1) helps remodel and stabilize primitive vasculature (Sato et al. 1993; Suri et al. 1996). The phenotype of mice lacking another Tie2 ligand, Ang2, suggested that Tie2 also functions during postnatal lymphatic remodeling (Gale et al. 2002); however, its expression has not been observed in LECs (Motoike et al. 2000; Saban et al. 2004; Wilting et al. 2006), except in restricted regions of adult lymphatics (Morisada et al. 2005; Tammela et al. 2005). Hence, Tie2-independent, integrin-mediated signaling might mediate angiopoietin function in the lymphatic vasculature (Morisada et al. 2005).

Our in situ hybridization and immunohistochemical (GFP) analyses of sections from E11.5, E13.5, and E15.5 Tie2-GFP transgenic mouse embryos failed to detect Tie2 expression in budding LECs, lymph sacs, developing lymphatics, or mesenchymal cells (Supplementary 
Fig. 6; data not shown). Furthermore, flow cytometric analysis failed to detect Tie $2^{+}$LECs in dissociated cells from E12.5 Tie2-GFP embryos (Supplementary Fig. 6B). Therefore, we reasoned that Tie 2 expression in BECs of the cardinal vein and its absence from embryonic LECs and mesenchymal cells support using the Tie2-Cre strain (Kisanuki et al. 2001) for cell-lineage analysis. Tie2-Cre mice were crossed with the $R 26 R$ reporter line, and embryos were isolated at different stages. At E11.5, Prox $1^{+}$ cells in the anterior cardinal vein and those budding from it were $1 a c Z^{+}$(Fig. 4A). Similarly, all E13.5 (Fig. 4B) and E14.5 (data not shown) Prox $1^{+}$ECs in the lymph sacs were $l a c Z^{+}$. These results support the proposal that mammalian lymph sacs are venous-derived.

Tie2-Cre;Prox $1^{\text {flox/+ }}$ mice We previously demonstrated that deletion of Prox1 from a conditional mouse strain (Tie2-Cre;Prox $1^{\text {flox/++}) ~(H a r v e y ~ e t ~ a l . ~ 2005) ~ r e s u l t e d ~ i n ~ c o n-~}$ ditional-heterozygous mice with lymphatic defects similar to those of standard Prox $1^{+/ L a c Z}$ mice (Wigle and Oliver 1999). This result suggested that early deletion of Prox1 from LEC progenitors in embryonic veins caused those lymphatic defects (Harvey et al. 2005). We used Tie2-Cre to generate conditional Prox1-null embryos (Tie2-Cre;Prox $1^{\text {flox/LacZ }) . ~ W e ~ r e a s o n e d ~ t h a t ~ i f ~ t h e ~ l y m-~}$ phatic vasculature is exclusively venous derived, lymphangiogenesis in the conditional-null embryos should be severely compromised. To this end, Prox $1^{+/ L a c Z} \mathrm{fe}-$ males were bred with Tie2-Cre;Prox $1^{\text {flox/+ }}$ males, and E11.5 Tie2-Cre;Prox $1^{\text {flox/LacZ }}$-null embryos were sectioned and analyzed using anti-Proxl antibody. At this stage, wild-type and Prox $1^{+/ L a c Z}$ embryos showed Prox $1^{+}$ LECs budding from the anterior cardinal vein (Fig. 5A); however, the conditional Tie2-Cre;Prox flox/LacZ mutant embryos showed very few Proxl-expressing LECs in or around the anterior cardinal vein (Fig. 5B). Similarly, whole-mount X-gal staining of E15.5 conditional Tie2-Cre;Prox $1^{\text {flox/LacZ }}$ mutant embryos revealed the presence of occasional scattered superficial $1 a c Z^{+}$LECs (Fig. 5D); many of the remaining superficial $\mathrm{lac}^{+}$cells were not LECs as they are also detected in E14.5 standard Prox1-null embryos (Supplementary Fig. 7, arrows; Wigle and Oliver 1999|. We must emphasize that using Cre lines results in variable efficiency in deletion of the gene of interest. As a consequence, the efficiency of deleting Proxl-expressing LEC progenitors in the cardinal vein was variable among embryos, and occasional superficial lac $Z^{+}$LECs were identified in the conditional mutant embryos. Importantly, no deep lymphatic vasculature was identified (Fig. 5E,F); only a few occasional scattered $1 a c Z^{+}$LECs were visualized.

Together, the defects in lymphatic patterning and integrity observed in conditional Tie2-Cre;Prox $1^{\text {flox/+ }}$-heterozygous mice (Harvey et al. 2005) and the drastic reduction in the number of LECs present in the conditional Tie2-Cre;Prox $1^{\text {flox/LacZ }}$ mutant embryos further validate our argument that in the developing mouse Prox1-expressing venous ECs are the major (if not the sole) source of LEC progenitors.

Tie2-Cre;Coup-TFII flox/+ mice Coup-TFII is an orphan nuclear receptor transcription factor, and in its absence, mouse embryos die around E10.0 due to several defects, including abnormal venous development (Pereira et al. 1999). Conditional deletion of Coup-TFII from embryonic veins causes abnormal venous expression of arterial markers and the subsequent death of embryos at around E11.0 (You et al. 2005). We speculated that if veins are the single source of LEC progenitors, the change in fate of venous ECs in Coup-TFII-mutant embryos should affect lymphatic development. Therefore, we conditionally deleted Coup-TFII from the vascular system of E11.0 embryos by using the Tie2-Cre strain. This deletion activated a lacZ reporter gene (Takamoto et al. 2005), allowing us to follow the lineage of Coup-TFII-expressing venous ECs. As controls, E11.0 conditional Coup-TFIIheterozygous (Tie2-Cre;Coup-TFIIflox/+ ) embryos were used.

Normally at E11.0, Prox1-expressing cells are detected in and budding from the anterior cardinal vein (Fig. 6A). Tie2-Cre;Coup-TFII flox/flox embryos had $\sim 80 \%$ fewer Prox1-expressing LECs in and around the cardinal vein than did their heterozygous littermates (Fig. 6B,C). Furthermore, the lymphatic marker Lyve-1 (Banerji et al.

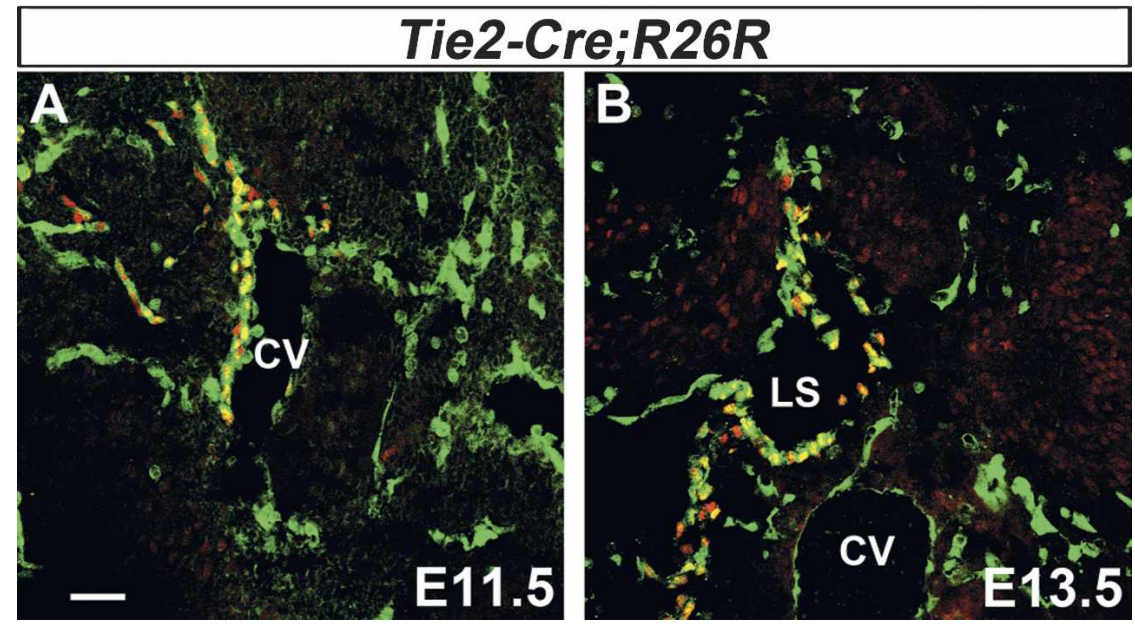

Figure 4. Lineage tracing using the BECspecific Tie2-Cre strain reveals the venous origin of LECs. Transverse sections of Tie2-Cre;R26R embryos were analyzed using antibodies against Prox1 (red) and $\beta$-gal (green) at E11.5 (A) and E13.5 (B). Nearly all Prox $1^{+}$cells in and around the anterior cardinal vein $(\mathrm{CV})$ colocalized with $\beta$-gal, which indicated that they originated from $\mathrm{Tie}^{+}$venous ECs. (LS) Lymph sac. Bar, $100 \mu \mathrm{m}$. 

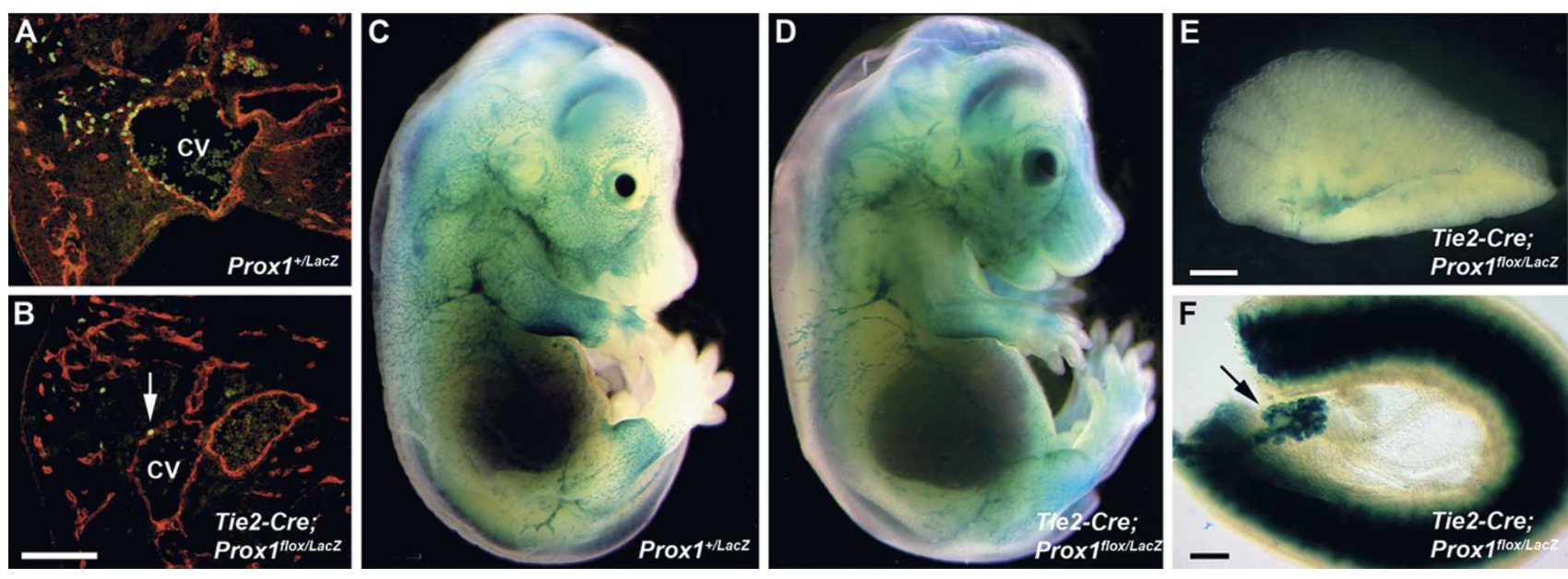

Figure 5. Conditional deletion of Prox1-expressing venous progenitors severely reduces the number of LECs. $(A)$ Transverse section of an E11.5 Prox $1^{+/ L a c Z}$ embryo showing normal Prox1 expression (green) in LECs located in and around the cardinal vein (CV). (B) The number of Prox1-expressing LECs in a Tie2-Cre;Prox $1^{\text {flox } / \mathrm{LacZ}}$ conditional mutant littermate was severely reduced in (arrow) and around the CV. The presence of a few remaining Prox $1^{+}$cells in the CV suggests that the Cre-mediated deletion of Prox 1 was incomplete. Red staining labels PECAM. $(C, D)$ The drastic reduction in the number of LEC progenitors affected the overall development of the lymphatic vasculature at later stages. $(C)$ The network of superficial lymphatics is vast in an X-gal-stained E15.5 control Prox $1^{+/ L a c Z}$ embryo. $(D)$ Instead, only a few scattered lac $Z^{+}$LECs are detected in a conditional Tie2-Cre; Prox $1^{\text {flox } / \text { LacZ }}$ mutant embryo.

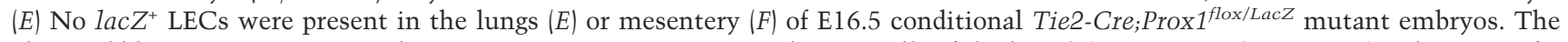
observed blue staining corresponds to Proxl expression in neuroendocrine cells of the lung $(E)$ or pancreas (arrow in F) and nonspecific trapping in the intestinal tract $(F)$. Bars: $A, B, 100 \mu \mathrm{m} ; E, 500 \mu \mathrm{m} ; F, 200 \mu \mathrm{m}$.

1999), which is normally expressed in the anterior cardinal vein at this stage, was absent in the mutant embryos (data not shown), supporting the proposal that venous identity is a necessary prerequisite for LEC specification in the cardinal veins.

The presence of Prox1-expressing cells in the conditional Coup-TFII-null embryos was probably the consequence of inefficient Cre activity and, thus, of residual venous identity as previously reported (You et al. 2005). This conclusion is supported by the fact that most of the Prox $1^{+}$cells in and around the cardinal vein in the E11.0 conditional Coup-TFII-heterozygous or -null embryos were also $\operatorname{lacZ}^{+}$(data not shown), which indicates that they originated from the vein. These results indicate that venous identity is required for embryonic lymphangiogenesis and that Proxl-expressing venous ECs are the main source of LECs in the developing mammalian embryo. However, a contribution of hematopoietic-derived LEC progenitors to the developing lymphatics has also been suggested (Buttler et al. 2006; Sebzda et al. 2006). Therefore, we further addressed whether hematopoieticderived LECs (lymphangioblasts) contribute to the mammalian embryonic lymphatic vasculature in another mouse model.
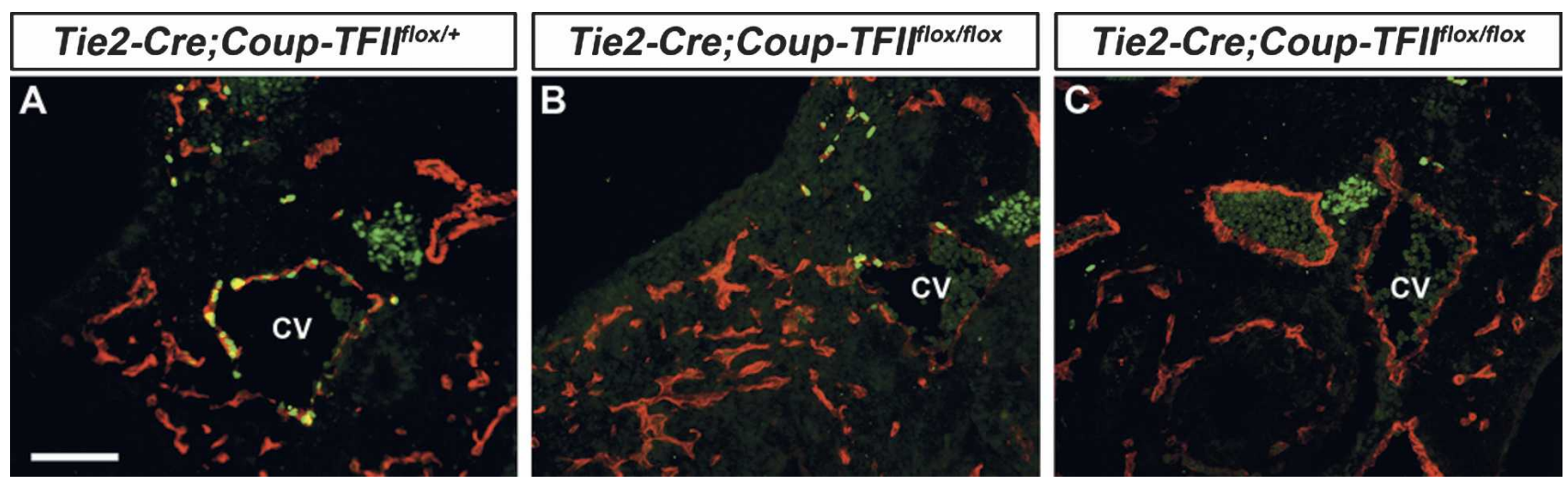

Figure 6. Defective venous identity compromises lymphatic vasculature development. (A) Prox1-expressing LECs (green) are seen in and around the anterior cardinal vein $(\mathrm{CV})$ in transverse sections of control E11.0 Tie2-Cre;Coup-TFII flox/+ mice. The number of Proxl-expressing LECs is drastically reduced $(B)$ or almost undetected $(C)$ in conditional Tie2-Cre;Coup-TFII flox/flox mutant littermates. Red staining labels PECAM. Bar, $100 \mu \mathrm{m}$. 
Runx1 mice Definitive hematopoiesis is defective in Runx1 mice, and Runx1-null mice die at around E12.5 (Okuda et al. 1996), when the first lymph sacs form. We hypothesized that if hematopoietic precursors contribute to the lymphatic vasculature, then initial stages of LEC budding and lymph sac formation should be affected in E12.5 Runx1-null embryos. If the sacs appeared normal, then hematopoietic precursors make little or no contribution to lymphangiogenesis.

In E12.0 Runx1 $1^{-/}$embryos labeled with anti-Lyve-1 antibodies, morphologically normal lymph sacs formed, but were blood-filled (Fig. 7B). Defects in lympho-venous separation may have caused this phenotype as SIp76 and Syk, which are expressed in hematopoietic cells, are essential for lympho-venous separation (Abtahian et al. 2003; Sebzda et al. 2006).
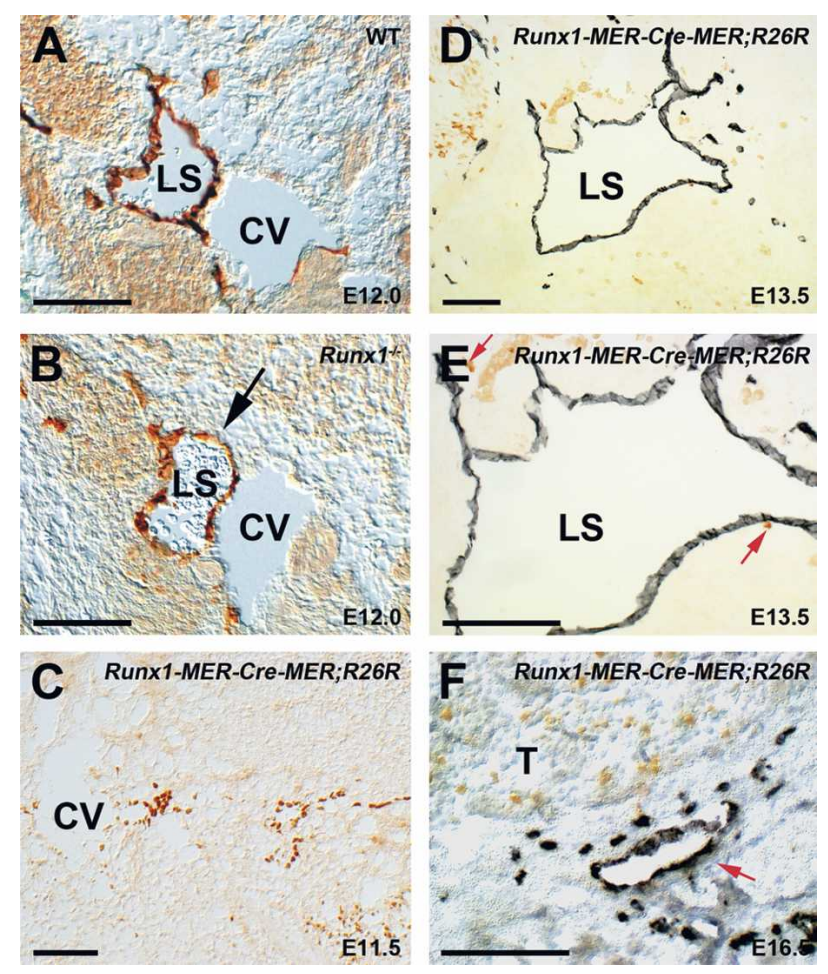

Figure 7. Hematopoietic cells do not contribute to the lymphatic vasculature. (A) Transverse section $(10 \mu \mathrm{m})$ of an E12.0 wild-type embryo showing a Lyve- $1^{+}$jugular lymph sac (LS) beside the anterior cardinal vein $(\mathrm{CV})$. (B) Transverse section of a Run $\times 1^{-/-}$littermate showing no obvious difference in the appearance of the jugular lymph sac, but the sac is blood-filled (arrow). Runx1-MER-Cre-MER;R26R embryos exposed to TM at E9.5 were analyzed at E11.5 $(C)$, E13.5 $(D, E)$, and E16.5 $(F) .(C)$ An X-gal-stained E11.5 embryo was sectioned and immunostained for Proxl (brown). No blue cells (descendents of Runx1 ${ }^{+}$ cells) are observed in the field. $(D, E)$ Lower- and higher-magnification pictures of a section from an E13.5 embryo coimmunostained for $\beta$-gal (brown) and Lyve-1 (purple). $\beta$-gal ${ }^{+}$cells (red arrows) can be seen around the lymph sacs (LS) but do not colocalize with Lyve-1 staining. (F) E16.5 embryo coimmunostained for $\beta$-gal (brown) and Lyve-1 (purple). $\beta$-Gal ${ }^{+}$cells are seen in the thymus $(\mathrm{T})$ but not in the lymphatic vessel (arrow). Bar, $100 \mu \mathrm{m}$.
Runx1-MER-Cre-MER mice A TM-inducible Cre line under the control of the Runx1 promoter was recently generated (Samokhvalov et al. 2007). Uniform labeling of the descendants of all definitive hematopoietic stem cells was achieved by crossing this strain with the $R 26 R$ reporter line and administering TM at E9.5 (Samokhvalov et al. 2007). Following this approach, we analyzed in detail E11.5, E13.5, and E16.5 Runx1-MER-Cre$M E R ; R 26 R$ embryos exposed to $5 \mathrm{mg}$ of TM at E9.5 (Fig. 7C-F; Supplementary Fig. 8). The lac $Z^{+}$cells were randomly distributed throughout the embryos; some were located around and occasionally in contact with the lymph sacs (Fig. 7D,E). None of the lacZ $^{+}$cells were double-labeled for any LEC-specific marker (Fig. 7C-F). This result agrees with other reports of early segregation of the hematopoietic compartment and EC compartment with no contribution of the former to the latter (Stadtfeld and Graf 2005; Samokhvalov et al. 2007).

Regarding the blood-filled lymph sacs observed in E12.0 Runx $1^{-/-}$embryos, the lineage-tracing analysis performed using the generated Runx1-MER-Cre-MER; $R 26 R$ embryos ruled out a cell-autonomous mechanism as being responsible for that phenotype. In fact, work performed using Runx $1^{-/-}$mice has previously demonstrated that hematopoietic stem cells regulate angiogenesis in a non-cell-autonomous manner (Takakura et al. 2000). Accordingly, the blood-filled lymph sac phenotype is most likely secondary to a primary angiogenic defect. Interestingly, angiogenic defects (abnormal artero-venous shunting) are also present in $S 1 p-76^{-/-}$embryos displaying a blood-filled lymphatic phenotype (Abtahian et al. 2003).

We conclude that hematopoietic-derived cells do not significantly contribute to the development of embryonic lymph sacs or to the formation of the lymphatic vasculature. Therefore, veins are the main if not sole source of LECs in the developing mammalian embryo.

\section{Discussion}

Here we conclusively demonstrated that in the developing mammalian embryo, Prox1-expressing LEC progenitors are of venous origin. Using detailed lineage-tracing analyses, we not only validated Sabin's original proposal (Sabin 1902) but also van der Putte's anatomical descriptions (van der Putte 1975). We corroborated that the rostral lymphatics form first and arise from the earliest Prox1-expressing LEC progenitors located in the anterior cardinal vein; the more posterior lymphatics form later from LEC progenitors in the posterior veins. Furthermore, we determined that local lymph sacs are the source of LECs that by sprouting, proliferation, and migration give rise to the lymphatic vasculature of nearby tissues and organs. The jugular lymph sacs give rise to the lymphatics of the lungs and most likely other nearby tissues; the posterior lymph sacs give rise to the lymphatics of the mesentery and posterior organs.

Using lineage tracing, we also determined that no descendants of Prox $1^{+}$cells were present in embryos exposed to TM prior to E9.75, thereby conclusively dem- 
onstrating that at around E9.75, ECs in the anterior cardinal vein are the earliest source of Proxl-expressing LEC progenitors. To demonstrate that the mammalian lymphatic vasculature has a solely venous origin, we used several mouse strains.

Tie2-Cre;Coup-TFIIflox/flox -mutant embryos showed that venous identity is required at least during the early stages of lymphatic development. Conditional Tie2Cre;Prox $1^{\text {flox/LacZ }}$ mutant embryos showed that the number of LECs was significantly reduced and that venous-derived Tie2 progeny contribute to the forming lymphatics. These results support the argument that LECs have a venous origin and that Proxl-expressing venous ECs are the main (if not the sole) source of LEC progenitors in developing mice.

Although our results show that developing lymphatic vasculature arises mostly from venous-derived Prox 1-expressing progenitor cells, two recent reports have claimed that other cell types contribute to developing lymphatics in mice. Buttler et al. (2006) proposed that scattered mesenchymal cells with leukocyte and lymphoendothelial characteristics that are first detected after E10.5 eventually integrate into the lymphatics. Sebzda et al. (2006) identified a subpopulation of Syk- and Slp-76-expressing hematopoietic-derived CEPs that acquire a lymphatic fate. However, two other reports found no evidence of hematopoietic contribution to the mammalian endothelial compartment (Stadtfeld and Graf 2005; Samokhvalov et al. 2007).

We did not identify descendents of Prox $1^{+}$cells in embryos exposed to TM prior to E9.5. This result not only demonstrated that E9.75 venous Prox1-expressing ECs are the earliest source of LEC progenitors but also eliminated the possibility that other hematopoietic- or mesenchyme-derived Prox $1^{+}$lymphatic progenitors are present before Proxl expression in the cardinal vein. Furthermore, Runx $1^{-/-}$embryos defective in definitive hematopoiesis had morphologically normal lymph sacs, and lineage tracing using the Runx1-MER-Cre-MER strain demonstrated that descendents of Runx $1^{+}$cells do not contribute to the developing lymph sacs. These results conclusively demonstrate that hematopoietic cells do not significantly contribute to the forming lymph sacs or lymphatic vasculature. Therefore, if mesenchymal cells with leukocyte and lymphoendothelial characteristics or hematopoietic-derived CEPs exist in the mouse embryo, they are so rare they probably cannot directly contribute to the developing murine lymphatic network. We must emphasize that additional LEC sources such as transdifferentiating macrophages (Maruyama et al. 2005) and bone marrow (Salven et al. 2003; Religa et al. 2005; Kerjaschki et al. 2006) probably contribute to postnatal lymphangiogenesis that occurs in normal and pathological conditions.

In summary, our work validates Sabin's model, which almost 100 years ago proposed that from venous-derived primary lymph sacs, the peripheral lymphatic system originates and spreads by endothelial sprouting into the surrounding tissues and organs, where capillaries form (Sabin 1902, 1904). We conclusively determined that during mammalian embryonic lymphangiogenesis, Proxl-expressing LEC progenitors in early veins are the main, and most likely unique source of LECs required for the formation of the lymphatic vasculature.

\section{Materials and methods}

Mice

Tie2-Cre mice were provided by Dr. M. Yanagisawa (University of Texas Southwestern Medical Center, Dallas, TX); Tie2-GFP mice were supplied by Dr. T. Sato (Cornell University, New York); R26R mice were provided by Dr. G. Grosveld (St. Jude Children's Research Hospital, Memphis, TN); and Runx1-mutant embryos were supplied by Dr. J. Downing (St. Jude Children's Research Hospital, Memphis, TN). The methods for generating Prox $1^{+/ L a c Z}$, Prox $1^{\text {flox/flox }}$, Coup-TFII flox/flox , and Runx1$M E R-C r e-M E R$ mice have been reported previously (Wigle et al. 1999; Harvey et al. 2005; Takamoto et al. 2005; Samokhvalov et al. 2007). To generate the Prox1-CreER ${ }^{T 2}$ embryonic stem cell line, we fused a synthetic splice acceptor site, Prox 1 exons 3 and 4, an internal ribosome entry site, and Cre-ER $R^{T 2}$ and Poly(A) tail. This fusion was targeted for insertion into intron 2 of the mouse Prox 1 locus by electroporation into the W9.5 embryonic stem cell line. Following selection and standard screening, the correctly targeted cells were used to generate chimeric mice. The developmental stage of mouse embryos was determined by considering 9 a.m. of the day the vaginal plug was detected in the pregnant dam as E0.5. All of the mouse experiments were approved by the St. Jude Children's Research Hospital Animal Care and Use Committee.

\section{Immunohistochemistry}

Fluorescent or horseradish peroxidase staining using 3,3' -diaminobenzidene $(\mathrm{DAB})$ as a substrate was performed on frozen or paraffin-embedded sections $(10 \mu \mathrm{m})$ as described previously (Harvey et al. 2005). Primary antibodies were rabbit anti- $\beta$-gal (MP Biomedicals), rabbit (AngioBio), and guinea pig (G. Oliver, unpubl.); anti-mouse Prox1; rat anti-mouse PECAM (BD Pharmingen); guinea pig anti-Lyve-1 (G. Oliver, unpubl.); and rabbit anti-GFP (Molecular Probes). Secondary antibodies were Alexa 488-conjugated donkey anti-rabbit (Molecular Probes), Cy3-conjugated donkey anti-guinea pig (Jackson ImmunoResearch Laboratories), and Cy3-conjugated donkey anti-rat (Jackson ImmunoResearch Laboratories).

\section{TM injection}

For lineage tracing using the Prox1-CreER ${ }^{T 2}$ line, TM $(20 \mathrm{mg} /$ $\mathrm{mL}$; Sigma) was dissolved in corn oil. Pregnant mice were injected intraperitoneally with either 3 or $5 \mathrm{mg}$ per $40 \mathrm{~g}$ of body weight at the indicated time points. Lineage tracing using the Runx1-MER-Cre-MER line has been described previously (Samokhvalov et al. 2007).

\section{Detection of $\beta$-galactosidase activity in embryos and tissues}

To detect $\beta$-gal activity in embryos, we performed X-gal staining as described previously (Harvey et al. 2005). Embryos were postfixed in $4 \%$ paraformaldehyde overnight at $4{ }^{\circ} \mathrm{C}$ and then cleared by soaking in sequentially increasing concentrations of glycerol dissolved in a solution of PBS and $0.1 \%$ Tween 20. Alternatively, embryos were embedded in paraffin and sectioned $(10 \mu \mathrm{m})$. 


\section{Acknowledgments}

We thank N. Lenny and J. Downing for Runx1-mutant embryos; M. Yanagisawa for the Tie2-Cre line; T. Sato for the Tie2-GFP; D. Dumont for the Tie2 cDNA; N. Gale, N. Papadopoulos, and G. Yancopoulos for the Lyve-1 fusion protein; M. Self and X. Geng for assistance; N. Johnson for critical reading; and Angela McArthur for editing the manuscript. This work was supported by NIH grant HL 076448 (to S.Y.T.) and R01-HL073402 (to G.O.), Cancer Center Support Grant CA-21765, and the American Lebanese Syrian Associated Charities (ALSAC). R.S.S. is supported by a post-doctoral fellowship grant from the Lymphatic Research Foundation.

\section{References}

Abtahian, F., Guerriero, A., Sebzda, E., Lu, M.M., Zhou, R., Mocsai, A., Myers, E.E., Huang, B., Jackson, D.G., Ferrari, V.A., et al. 2003. Regulation of blood and lymphatic vascular separation by signaling proteins SLP-76 and Syk. Science 299: 247-251.

Banerji, S., Ni, J., Wang, S.X., Clasper, S., Su, J., Tammi, R., Jones, M., and Jackson, D.G. 1999. LYVE-1, a new homologue of the CD44 glycoprotein, is a lymph-specific receptor for hyaluronan. J. Cell Biol. 144: 789-801.

Buttler, K., Kreysing, A., von Kaisenberg, C.S., Schweigerer, L., Gale, N., Papoutsi, M., and Wilting, J. 2006. Mesenchymal cells with leukocyte and lymphendothelial characteristics in murine embryos. Dev. Dyn. 235: 1554-1562.

Danielian, P.S., Muccino, D., Rowitch, D.H., Michael, S.K., and McMahon, A.P. 1998. Modification of gene activity in mouse embryos in utero by a tamoxifen-inducible form of Cre recombinase. Curr. Biol. 8: 1323-1326.

Dor, Y., Brown, J., Martinez, O.I., and Melton, D.A. 2004. Adult pancreatic $\beta$-cells are formed by self-duplication rather than stem-cell differentiation. Nature 429: 41-46.

Gale, N.W., Thurston, G., Hackett, S.F., Renard, R., Wang, Q., McClain, J., Martin, C., Witte, C., Witte, M.H., Jackson, D., et al. 2002. Angiopoietin-2 is required for postnatal angiogenesis and lymphatic patterning, and only the latter role is rescued by Angiopoietin-1. Dev. Cell 3: 411-423.

Harvey, N.L., Srinivasan, R.S., Dillard, M.E., Johnson, N.C., Witte, M.H., Boyd, K., Sleeman, M.W., and Oliver, G. 2005. Lymphatic vascular defects promoted by Prox1 haploinsufficiency cause adult-onset obesity. Nat. Genet. 37: 10721081.

Heuer, G. 1909. The development of the lymphatics in the small intestine of the pig. Am. J. Anat. 9: 93-118. doi: 10.1002/aja.1000090105.

Huntington, G.S. and McClure, C.F.W. 1910. The anatomy and development of the jugular lymph sac in the domestic cat (Felis domestica). Am. J. Anat. 10: 177-312. doi: 10.1002/ aja.1000100108.

Indra, A.K., Warot, X., Brocard, J., Bornert, J.M., Xiao, J.H., Chambon, P., and Metzger, D. 1999. Temporally-controlled site-specific mutagenesis in the basal layer of the epidermis: Comparison of the recombinase activity of the tamoxifeninducible Cre-ER(T) and Cre-ER(T2) recombinases. Nucleic Acids Res. 27: 4324-4327.

Kerjaschki, D., Huttary, N., Raab, I., Regele, H., Bojarski-Nagy, K., Bartel, G., Krober, S.M., Greinix, H., Rosenmaier, A., Karlhofer, F., et al. 2006. Lymphatic endothelial progenitor cells contribute to de novo lymphangiogenesis in human renal transplants. Nat. Med. 12: 230-234.

Kisanuki, Y.Y., Hammer, R.E., Miyazaki, J., Williams, S.C., Richardson, J.A., and Yanagisawa, M. 2001. Tie2-Cre trans- genic mice: A new model for endothelial cell-lineage analysis in vivo. Dev. Biol. 230: 230-242.

Maruyama, K., Ii, M., Cursiefen, C., Jackson, D.G., Keino, H., Tomita, M., Van Rooijen, N., Takenaka, H., D'Amore, P.A., Stein-Streilein, J., et al. 2005. Inflammation-induced lymphangiogenesis in the cornea arises from CD11b-positive macrophages. J. Clin. Invest. 115: 2363-2372.

Morisada, T., Oike, Y., Yamada, Y., Urano, T., Akao, M., Kubota, Y., Maekawa, H., Kimura, Y., Ohmura, M., Miyamoto, T., et al. 2005. Angiopoietin-1 promotes LYVE-1-positive lymphatic vessel formation. Blood 105: 4649-4656.

Motoike, T., Loughna, S., Perens, E., Roman, B.L., Liao, W., Chau, T.C., Richardson, C.D., Kawate, T., Kuno, J., Weinstein, B.M., et al. 2000. Universal GFP reporter for the study of vascular development. Genesis 28: 75-81.

Ny, A., Koch, M., Schneider, M., Neven, E., Tong, R.T., Maity, S., Fischer, C., Plaisance, S., Lambrechts, D., Heligon, C., et al. 2005. A genetic Xenopus laevis tadpole model to study lymphangiogenesis. Nat. Med. 11: 998-1004.

Okuda, T., van Deursen, J., Hiebert, S.W., Grosveld, G., and Downing, J.R. 1996. AML1, the target of multiple chromosomal translocations in human leukemia, is essential for normal fetal liver hematopoiesis. Cell 84: 321-330.

Oliver, G. and Alitalo, K. 2005. The lymphatic vasculature: Recent progress and paradigms. Annu. Rev. Cell Dev. Biol. 21: 457-483.

Oliver, G. and Detmar, M. 2002. The rediscovery of the lymphatic system: Old and new insights into the development and biological function of the lymphatic vasculature. Genes \& Dev. 16: 773-783.

Pereira, F.A., Qiu, Y., Zhou, G., Tsai, M.J., and Tsai, S.Y. 1999. The orphan nuclear receptor COUP-TFII is required for angiogenesis and heart development. Genes \& Dev. 13: 10371049.

Religa, P., Cao, R., Bjorndahl, M., Zhou, Z., Zhu, Z., and Cao, Y. 2005. Presence of bone marrow-derived circulating progenitor endothelial cells in the newly formed lymphatic vessels. Blood 106: 4184-4190.

Saban, M.R., Memet, S., Jackson, D.G., Ash, J., Roig, A.A., Israel, A., and Saban, R. 2004. Visualization of lymphatic vessels through NF-кB activity. Blood 104: 3228-3230.

Sabin, F. 1902. On the origin of the lymphatics system from the veins and the development of the lymph hearts and the thoracic duct in the pig. Am. J. Anat. 1: 367-389. doi: 10.1002/ aja. 1000010310.

Sabin, F. 1904. On the development of superficial lymphatics in the skin of the pig. Am. J. Anat. 3: 183-195. doi: 10.1002/ aja.1000030205.

Salven, P., Mustjoki, S., Alitalo, R., Alitalo, K., and Rafii, S. 2003. VEGFR-3 and CD133 identify a population of CD $34^{+}$ lymphatic/vascular endothelial precursor cells. Blood 101: 168-172.

Samokhvalov, I.M., Samokhvalova, N.I., and Nishikawa, S. 2007. Cell tracing shows the contribution of the yolk sac to adult haematopoiesis. Nature 446: 1056-1061.

Sato, T.N., Qin, Y., Kozak, C.A., and Audus, K.L. 1993. Tie-1 and tie-2 define another class of putative receptor tyrosine kinase genes expressed in early embryonic vascular system. Proc. Natl. Acad. Sci. 90: 9355-9358.

Sebzda, E., Hibbard, C., Sweeney, S., Abtahian, F., Bezman, N., Clemens, G., Maltzman, J.S., Cheng, L., Liu, F., Turner, M., et al. 2006. Syk and Slp-76 mutant mice reveal a cell-autonomous hematopoietic cell contribution to vascular development. Dev. Cell 11: 349-361.

Soriano, P. 1999. Generalized lacZ expression with the ROSA26 Cre reporter strain. Nat. Genet. 21: 70-71. 
Stadtfeld, M. and Graf, T. 2005. Assessing the role of hematopoietic plasticity for endothelial and hepatocyte development by non-invasive lineage tracing. Development 132: 203-213.

Suri, C., Jones, P.F., Patan, S., Bartunkova, S., Maisonpierre, P.C., Davis, S., Sato, T.N., and Yancopoulos, G.D. 1996. Requisite role of angiopoietin-1, a ligand for the TIE2 receptor, during embryonic angiogenesis. Cell 87: 1171-1180.

Takakura, N., Huang, X.L., Naruse, T., Hamaguchi, I., Dumont, D.J., Yancopoulos, G.D., and Suda, T. 1998. Critical role of the TIE2 endothelial cell receptor in the development of definitive hematopoiesis. Immunity 9: 677-686.

Takakura, N., Watanabe, T., Suenobu, S., Yamada, Y., Noda, T., Ito, Y., Satake, M., and Suda, T. 2000. A role for hematopoietic stem cells in promoting angiogenesis. Cell 102: 199-209.

Takamoto, N., You, L.R., Moses, K., Chiang, C., Zimmer, W.E., Schwartz, R.J., DeMayo, F.J., Tsai, M.J., and Tsai, S.Y. 2005. COUP-TFII is essential for radial and anteroposterior patterning of the stomach. Development 132: 2179-2189.

Tammela, T., Saaristo, A., Lohela, M., Morisada, T., Tornberg, J., Norrmen, C., Oike, Y., Pajusola, K., Thurston, G., Suda, T., et al. 2005. Angiopoietin-1 promotes lymphatic sprouting and hyperplasia. Blood 105: 4642-4648.

van der Putte, S.C. 1975. The early development of the lymphatic system in mouse embryos. Acta Morphol. Neerl. Scand. 13: 245-286.

Wigle, J.T. and Oliver, G. 1999. Proxl function is required for the development of the murine lymphatic system. Cell 98: 769-778.

Wigle, J.T., Chowdhury, K., Gruss, P., and Oliver, G. 1999. Prox 1 function is crucial for mouse lens-fibre elongation. Nat. Genet. 21: 318-322.

Wigle, J.T., Harvey, N., Detmar, M., Lagutina, I., Grosveld, G., Gunn, M.D., Jackson, D.G., and Oliver, G. 2002. An essential role for Proxl in the induction of the lymphatic endothelial cell phenotype. EMBO J. 21: 1505-1513.

Wilting, J., Papoutsi, M., Schneider, M., and Christ, B. 2000. The lymphatic endothelium of the avian wing is of somitic origin. Dev. Dyn. 217: 271-278.

Wilting, J., Aref, Y., Huang, R., Tomarev, S.I., Schweigerer, L., Christ, B., Valasek, P., and Papoutsi, M. 2006. Dual origin of avian lymphatics. Dev. Biol. 292: 165-173.

Yaniv, K., Isogai, S., Castranova, D., Dye, L., Hitomi, J., and Weinstein, B.M. 2006. Live imaging of lymphatic development in the zebrafish. Nat. Med. 12: 711-716.

You, L.R., Lin, F.J., Lee, C.T., DeMayo, F.J., Tsai, M.J., and Tsai, S.Y. 2005. Suppression of Notch signalling by the COUPTFII transcription factor regulates vein identity. Nature 435: 98-104.

Zhang, H., Fujitani, Y., Wright, C.V., and Gannon, M. 2005. Efficient recombination in pancreatic islets by a tamoxifeninducible Cre-recombinase. Genesis 42: 210-217. 


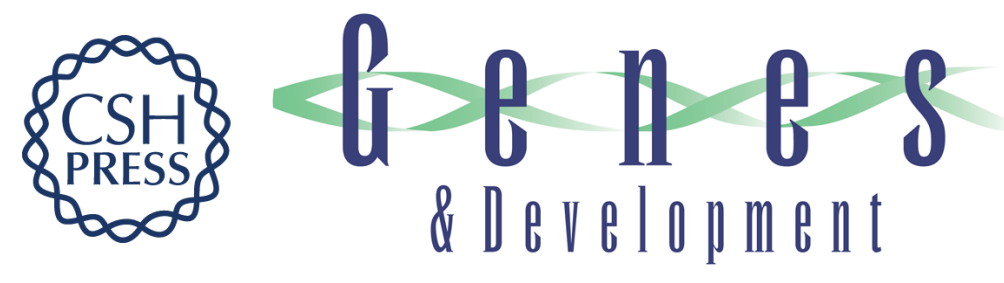

\section{Lineage tracing demonstrates the venous origin of the mammalian lymphatic vasculature}

R. Sathish Srinivasan, Miriam E. Dillard, Oleg V. Lagutin, et al.

Genes Dev. 2007, 21:

Access the most recent version at doi:10.1101/gad.1588407

Supplemental http://genesdev.cshlp.org/content/suppl/2007/10/01/21.19.2422.DC1
Material

References This article cites 44 articles, 13 of which can be accessed free at: http://genesdev.cshlp.org/content/21/19/2422.full.html\#ref-list-1

License

Email Alerting

Receive free email alerts when new articles cite this article - sign up in the box at the top Service right corner of the article or click here.

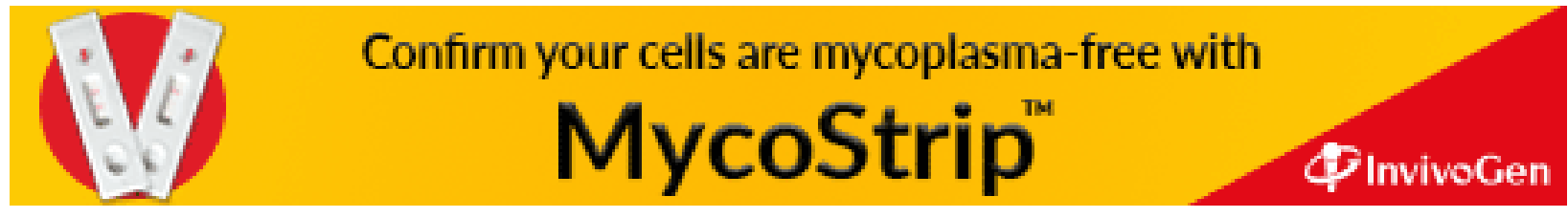

